\title{
Facet Connectedness of Discrete Hyperplanes with Zero Intercept: The General Case
}

\author{
Eric Domenjoud ${ }^{1}$, Xavier Provençal $^{2}$, and Laurent Vuillon ${ }^{2}$ \\ 1 CNRS, Loria, UMR CNRS 7503, Nancy, France \\ Eric.Domenjoud@loria.fr \\ 2 Université de Savoie, LAMA, UMR CNRS 5127, Chambéry, France \\ \{Xavier.Provencal, Laurent. Vuillon\}@univ-savoie.fr
}

\begin{abstract}
A digital discrete hyperplane in $\mathbb{Z}^{d}$ is defined by a normal vector $\mathbf{v}$, a shift $\mu$, and a thickness $\theta$. The set of thicknesses $\theta$ for which the hyperplane is connected is a right unbounded interval of $\mathbb{R}^{+}$. Its lower bound, called the connecting thickness of $\mathbf{v}$ with shift $\mu$, may be computed by means of the fully subtractive algorithm. A careful study of the behaviour of this algorithm allows us to give exhaustive results about the connectedness of the hyperplane at the connecting thickness in the case $\mu=0$. We show that it is connected if and only if the sequence of vectors computed by the algorithm reaches in finite time a specific set of vectors which has been shown to be Lebesgue negligible by Kraaikamp \& Meester.
\end{abstract}

Keywords: discrete hyperplane, connectedness, connecting thickness, fully subtractive algorithm.

\section{Preliminaries}

In order to prevent any ambiguity, we denote by $\mathbb{N}_{0}$ the set of nonnegative integers $\left(\mathbb{N}_{0}=\{0,1,2, \ldots\}\right)$, and by $\mathbb{N}_{1}$ the set of positive integers $\left(\mathbb{N}_{1}=\{1,2, \ldots\}\right)$. We denote by $\mathbb{R}^{+}$the set of non-negative real numbers. Given $d \in \mathbb{N}_{1},\left(\mathbf{e}_{1}, \ldots, \mathbf{e}_{d}\right)$ denotes the canonical basis of $\mathbb{R}^{d}$. The usual scalar product on $\mathbb{R}^{d}$ is denoted by $\langle.,$.$\rangle . For any vector \mathbf{v} \in \mathbb{R}^{d},\|\mathbf{v}\|_{1}$ and $\|\mathbf{v}\|_{\infty}$ denote respectively the usual 1-norm and $\infty$-norm of $\mathbf{v}$, which means $\|\mathbf{v}\|_{1}=\sum_{i=1}^{d}\left|v_{i}\right|$, and $\|\mathbf{v}\|_{\infty}=$ $\max _{i}\left|v_{i}\right|$. Given $\mathbf{v} \in \bigcup_{d \geqslant 1} \mathbb{R}^{d}$, we denote by $\# \mathbf{v}$ the dimension of the space $\mathbf{v}$ belongs to, that is to say, $\mathbf{v} \in \mathbb{R}^{\# \mathbf{v}}$. Given $\mathbf{v}=\left(v_{1}, \ldots, v_{d}\right) \in \mathbb{R}^{d}$ we denote by $\operatorname{dim}_{\mathbb{Q}}\left(v_{1}, \ldots, v_{d}\right)$, or simply by $\operatorname{dim}_{\mathbb{Q}}(\mathbf{v})$ the dimension of $v_{1} \mathbb{Q}+\cdots+v_{d} \mathbb{Q}$ as a vector space over $\mathbb{Q}$. If $\operatorname{dim}_{\mathbb{Q}}\left(v_{1}, \ldots, v_{d}\right)=1$ then we denote by $\operatorname{gcd}\left(v_{1}, \ldots, v_{d}\right)$, or simply by $\operatorname{gcd}(\mathbf{v})$, the greatest real number $\gamma$ such that $v_{i} / \gamma$ is an integer for all $i$. Two distinct points $\mathbf{x}$ and $\mathbf{y}$ in $\mathbb{Z}^{d}$ are facet-neighbours (neighbours for short) if $\|\mathbf{x}-\mathbf{y}\|_{1}=1$ or equivalently if $\mathbf{x}-\mathbf{y}= \pm \mathbf{e}_{i}$ for some $i \in\{1, \ldots, d\}$. This notion of neighbouring refers to the representation of a point in $\mathbb{Z}^{d}$ as a voxel, i.e as a unit cube centred at the point. Two points are facet-neighbours if the voxels representing them share a facet. A path in $\mathbb{Z}^{d}$ is a sequence $\left(\mathbf{x}^{1}, \ldots, \mathbf{x}^{n}\right)$

E. Barcucci et al. (Eds.): DGCI 2014, LNCS 8668, pp. 1-12 2014.

(c) Springer International Publishing Switzerland 2014 
such that $\mathbf{x}^{i-1}$ and $\mathbf{x}^{i}$ are neighbours for all $i \in\{2, \ldots, n\}$. A subset $S$ of $\mathbb{Z}^{d}$ is connected if it is not empty, and for all pairs of points $\mathbf{x}$ and $\mathbf{y}$ in $S$, there exists a path $\left(\mathbf{x}^{1}, \ldots, \mathbf{x}^{n}\right)$ in $S$ such that $\mathbf{x}^{1}=\mathbf{x}$ and $\mathbf{x}^{n}=\mathbf{y}$.

Given a vector $\mathbf{v} \in \mathbb{R}^{d} \backslash\{\mathbf{0}\}$, and two real numbers $\mu$ and $\theta$, the arithmetic discrete hyperplane with normal vector $\mathbf{v}$, shift $\mu$, and thickness $\theta$ 111, denoted by $\mathbb{P}(\mathbf{v}, \mu, \theta)$, is the subset of $\mathbb{Z}^{d}$ defined by

$$
\mathbb{P}(\mathbf{v}, \mu, \theta)=\left\{\mathbf{x} \in \mathbb{Z}^{d} \mid 0 \leqslant\langle\mathbf{v}, \mathbf{x}\rangle+\mu<\theta\right\} .
$$

Given a vector $\mathbf{v} \in \mathbb{R}^{d} \backslash\{\mathbf{0}\}$ and a shift $\mu \in \mathbb{R}$, we are interested in the set of values $\theta$ for which $\mathbb{P}(\mathbf{v}, \mu, \theta)$ is connected. This set is known to be a rightunbounded interval of $\mathbb{R}^{+}$5. Its lower bound is called the connecting thickness of $\mathbf{v}$ with shift $\mu$, and is denoted by $\Omega(\mathbf{v}, \mu)$. By definition, $\mathbb{P}(\mathbf{v}, \mu, \theta)$ is connected if $\theta>\Omega(\mathbf{v}, \mu)$, and disconnected if $\theta<\Omega(\mathbf{v}, \mu)$. The question that we address in this work is the connectedness of $\mathbb{P}(\mathbf{v}, \mu, \theta)$ at the critical thickness $\Omega(\mathbf{v}, \mu)$, i.e. whether $\mathbb{P}(\mathbf{v}, \mu, \Omega(\mathbf{v}, \mu))$ is connected or not. In most cases, it is easily shown that the answer is negative. Only for a specific class of vectors, the answer was unknown up to now, although some partial results have been established [23]. We present here the general case when $\mu=0$.

The problem of computing the connecting thickness has already been addressed several times 4568910 . This computation may be performed by means of the fully subtractive algorithm 12 . We recall it briefly in the next section, and by the way, we give some useful properties.

\section{Computation of the Connecting Thickness}

Let us start by giving some bounds on $\Omega(\mathbf{v}, \mu)$ which will be useful later.

Theorem 1 ([5]). Let $d \geqslant 1, \mathbf{v} \in \mathbb{R}^{d} \backslash\{\mathbf{0}\}$, and $\mu, \theta \in \mathbb{R}$.

- If $\mathbf{v}$ has exactly one non zero coordinate $v_{i}$, then $\mathbb{P}(\mathbf{v}, \mu, \theta)$ is connected if and only if $\theta>\mu \bmod \left|v_{i}\right|=\mu \bmod \operatorname{gcd}(\mathbf{v})$. Therefore, for all $\mu$, we have $\Omega(\mathbf{v}, \mu)=\mu \bmod \operatorname{gcd}(\mathbf{v})$.

- If $\mathbf{v}$ has at least two non zero coordinates then let $\xi(\mathbf{v})=\min \left\{\left|v_{i}\right| \mid v_{i} \neq 0\right\}$.

- If $\theta \leqslant\|\mathbf{v}\|_{\infty}$ then $\mathbb{P}(\mathbf{v}, \mu, \theta)$ is disconnected for all $\mu$.

- If $\theta \geqslant\|\mathbf{v}\|_{\infty}+\xi(\mathbf{v})$ then $\mathbb{P}(\mathbf{v}, \mu, \theta)$ is connected for all $\mu$.

Therefore, for all $\mu$, we have $\|\mathbf{v}\|_{\infty} \leqslant \Omega(\mathbf{v}, \mu) \leqslant\|\mathbf{v}\|_{\infty}+\xi(\mathbf{v})$.

The problem of computing the connecting thickness may first be simplified thanks to the following relation [5] which allows us to get rid of the shift $\mu$ in the computations:

$$
\Omega(\mathbf{v}, \mu)= \begin{cases}\Omega(\mathbf{v}, 0)+(\mu \bmod \operatorname{gcd}(\mathbf{v})) & \text { if } \operatorname{dim}_{\mathbb{Q}}(\mathbf{v})=1 \\ \Omega(\mathbf{v}, 0) & \text { if } \operatorname{dim}_{\mathbb{Q}}(\mathbf{v}) \geqslant 2\end{cases}
$$

We are then left to compute $\Omega(\mathbf{v}, 0)$ that we simply denote by $\Omega(\mathbf{v})$. For convenience, we shall usually write $\Omega\left(v_{1}, \ldots, v_{d}\right)$ instead of $\Omega\left(\left(v_{1}, \ldots, v_{d}\right)\right)$. The problem may be further simplified thanks to the observation that for any permutation 
$\sigma$ of $\{1, \ldots, d\}, \mathbb{P}(\mathbf{v}, \mu, \theta)$ is connected if and only if $\mathbb{P}\left(\left(\left|v_{\sigma(1)}\right|, \ldots,\left|v_{\sigma(d)}\right|\right), \mu, \theta\right)$ is connected [5. We may therefore assume that $\mathbf{v}$ belongs to $\left(\mathbb{R}^{+}\right)^{d} \backslash\{\mathbf{0}\}$, and that its coordinates are suitably ordered. In the sequel, we denote by $\mathcal{O}_{d}^{+}$the set of vectors $\mathbf{v} \in \mathbb{R}^{d} \backslash\{\mathbf{0}\}$ such that $0 \leqslant v_{1} \leqslant \cdots \leqslant v_{d}$. Finally, if $\mathbf{v} \in \mathcal{O}_{d}^{+}$and $v_{1}=0$, then $\mathbb{P}\left(\left(0, v_{2}, \ldots, v_{d}\right), \mu, \theta\right)$ is connected if and only if $\mathbb{P}\left(\left(v_{2}, \ldots, v_{d}\right), \mu, \theta\right)$ is connected.

The following theorem is the key to the computation of $\Omega(\mathbf{v})$. It appears under various forms and in various contexts in the literature.

Theorem $2\left(\left[\begin{array}{l}5,68,9] \\ \mathbf{1 0}\end{array}\right)\right.$. Let $d \geqslant 2, \mathbf{v} \in \mathcal{O}_{d}^{+}$, and $\mathbf{v}^{\prime}=\left(v_{1}, v_{2}-v_{1}, \ldots, v_{d}-\right.$ $\left.v_{1}\right)$. For all $\mu, \theta \in \mathbb{R}, \mathbb{P}(\mathbf{v}, \mu, \theta)$ is connected if and only if $\mathbb{P}\left(\mathbf{v}^{\prime}, \mu, \theta-v_{1}\right)$ is connected. Therefore, $\Omega(\mathbf{v}, \mu)=v_{1}+\Omega\left(\mathbf{v}^{\prime}, \mu\right)$.

We define $\pi$ and $\sigma$ as:

$$
\begin{aligned}
\pi: \bigcup_{d \geqslant 2} \mathbb{R}^{d} & \rightarrow \bigcup_{d \geqslant 2} \mathbb{R}^{d-1} \\
\left(v_{1}, v_{2}, \ldots, v_{d}\right) & \mapsto\left(v_{2}, \ldots, v_{d}\right) ; \\
\sigma: \bigcup_{d \geqslant 2} \mathbb{R}^{d} & \rightarrow \bigcup_{d \geqslant 2} \mathbb{R}^{d} \\
\left(v_{1}, v_{2}, \ldots, v_{d}\right) & \mapsto\left(v_{1}, v_{2}-v_{1}, \ldots, v_{d}-v_{1}\right) .
\end{aligned}
$$

Thanks to Th. 2 and to the preceding remarks, given $\mathbf{v} \in \mathcal{O}_{d}^{+}$, we may compute $\Omega(\mathbf{v})$ recursively as follows:

$$
\Omega(\mathbf{v})= \begin{cases}0 & \text { if } \# \mathbf{v}=1, \\ \Omega(\pi(\mathbf{v})) & \text { if } \# \mathbf{v} \geqslant 2 \text { and } v_{1}=0 \\ v_{1}+\Omega(\operatorname{sort}(\sigma(\mathbf{v}))) & \text { if } \# \mathbf{v} \geqslant 2 \text { and } v_{1}>0\end{cases}
$$

where 'sort' orders the coordinates of its argument in non decreasing order.

The algorithm deduced from these equations is known as the Ordered Fully Subtractive algorithm (OFS) [12. In effect, OFS computes a possibly infinite sequence of pairs $\left(\mathbf{v}^{n}, \Omega^{n}\right)_{n \geqslant 1}$ defined by:

$$
\begin{aligned}
& \left(\mathbf{v}^{1}, \Omega^{1}\right)=(\operatorname{sort}(\mathbf{v}), 0) \text { and for all } n \geqslant 1 \text { such that } \# \mathbf{v}^{n} \geqslant 2 \\
& \left(\mathbf{v}^{n+1}, \Omega^{n+1}\right)= \begin{cases}\left(\pi\left(\mathbf{v}^{n}\right), \Omega^{n}\right) & \text { if } v_{1}^{n}=0 \\
\left(\operatorname{sort}\left(\sigma\left(\mathbf{v}^{n}\right)\right), \Omega^{n}+v_{1}^{n}\right) & \text { if } v_{1}^{n}>0\end{cases}
\end{aligned}
$$

If $\# \mathbf{v}^{n}=1$ for some $n$, then OFS actually terminates, and the sequence is finite. This sequence has the following properties. For all $\theta, \mu \in \mathbb{R}$ and all $n \geqslant 1$ such that $\left(\mathbf{v}^{n}, \Omega^{n}\right)$ is defined:

- $\Omega^{n}=\sum_{i=1}^{n-1} v_{1}^{i}$

- $\Omega(\mathbf{v}, \mu)=\Omega^{n}+\Omega\left(\mathbf{v}^{n}, \mu\right)$;

- $\mathbb{P}(\mathbf{v}, \mu, \theta)$ is connected if and only if $\mathbb{P}\left(\mathbf{v}^{n}, \mu, \theta-\Omega^{n}\right)$ is connected;

- $\mathbb{P}(\mathbf{v}, \mu, \Omega(\mathbf{v}, \mu))$ is connected if and only if $\mathbb{P}\left(\mathbf{v}^{n}, \mu, \Omega\left(\mathbf{v}^{n}, \mu\right)\right)$ is connected. 
At each step of the algorithm, either $\mathbf{v}$ decreases componentwise, or the number of coordinates of $\mathbf{v}$ decreases, which may happen only finitely many times. Also, $\Omega^{n}$ is increasing and bounded by $\Omega(\mathbf{v})$. OFS is therefore convergent in the sense that $\mathbf{v}^{n}$ and $\Omega^{n}$ tend towards limits $\mathbf{v}^{\infty}$ and $\Omega^{\infty}$. However, it terminates if and only if $\operatorname{dim}_{\mathbb{Q}}\left(v_{1}, \ldots, v_{d}\right)=1$.

If OFS terminates, which means that at some step $n_{0}$, we have $\# \mathbf{v}^{n_{0}}=1$, then $\Omega(\mathbf{v})=\Omega^{n_{0}}$, and for all $\mu \in \mathbb{R}$,

$$
\Omega(\mathbf{v}, \mu)=\Omega^{n_{0}}+(\mu \bmod \operatorname{gcd}(\mathbf{v})) .
$$

If OFS does not terminate, then for all $\mu \in \mathbb{R}$, we have

$$
\Omega(\mathbf{v}, \mu)=\Omega(\mathbf{v})=\Omega^{\infty}+\left\|\mathbf{v}^{\infty}\right\|_{\infty} .
$$

Indeed, for all $n \geqslant 1$, we have $\Omega(\mathbf{v}, \mu)=\Omega^{n}+\Omega\left(\mathbf{v}^{n}, \mu\right)$, and, by Th. 1, we have $\left\|\mathbf{v}^{n}\right\|_{\infty} \leqslant \Omega\left(\mathbf{v}^{n}, \mu\right) \leqslant\left\|\mathbf{v}^{n}\right\|_{\infty}+\xi\left(\mathbf{v}^{n}\right)$. Since $\lim _{n \rightarrow \infty} \xi\left(\mathbf{v}^{n}\right)=0$, we get $\Omega(\mathbf{v}, \mu)=\lim _{n \rightarrow \infty}\left(\Omega^{n}+\Omega\left(\mathbf{v}^{n}, \mu\right)\right)=\Omega^{\infty}+\left\|\mathbf{v}^{\infty}\right\|_{\infty}$. In this case, there must exist $n_{0}$ such that $\# \mathbf{v}^{n} \geqslant 2$, and $v_{1}^{n}>0$ for all $n \geqslant n_{0}$. Let $d_{\infty}=\# \mathbf{v}^{n_{0}}$. Note that $d_{\infty} \geqslant 2$. Then for all $n \geqslant n_{0}$, we have $\left\|\mathbf{v}^{n+1}\right\|_{1}=\left\|\mathbf{v}^{n}\right\|_{1}-\left(d_{\infty}-1\right) v_{1}^{n}$, so that

$$
\Omega^{\infty}=\Omega^{n_{0}}+\sum_{n=n_{0}}^{\infty} v_{1}^{n}=\Omega^{n_{0}}+\sum_{n=n_{0}}^{\infty} \frac{\left\|\mathbf{v}^{n}\right\|_{1}-\left\|\mathbf{v}^{n+1}\right\|_{1}}{d_{\infty}-1}=\Omega^{n_{0}}+\frac{\left\|\mathbf{v}^{n_{0}}\right\|_{1}-\left\|\mathbf{v}^{\infty}\right\|_{1}}{d_{\infty}-1}
$$

For all $\mu \in \mathbb{R}$, we get

$$
\Omega(\mathbf{v}, \mu)=\Omega(\mathbf{v})=\Omega^{n_{0}}+\frac{\left\|\mathbf{v}^{n_{0}}\right\|_{1}-\left\|\mathbf{v}^{\infty}\right\|_{1}}{d_{\infty}-1}+\left\|\mathbf{v}^{\infty}\right\|_{\infty} .
$$

In particular, if $v_{1}^{n}>0$ for all $n$, then $n_{0}=1$ and $d_{\infty}=d$, so that

$$
\Omega(\mathbf{v})=\frac{\|\mathbf{v}\|_{1}-\left\|\mathbf{v}^{\infty}\right\|_{1}}{d-1}+\left\|\mathbf{v}^{\infty}\right\|_{\infty}
$$

Theorem $3\left([\mathbf{9}[\mathbf{1 0}])\right.$. Let $d \geqslant 2$ and $\mathbf{v} \in \mathcal{O}_{d}^{+}$. If $v_{1}^{n}>0$ for all $n$, then

$$
\lim _{n \rightarrow \infty} \mathbf{v}^{n}=\mathbf{0} \text { if and only if }\left\|\mathbf{v}^{n}\right\|_{\infty} \leqslant\left\|\mathbf{v}^{n}\right\|_{1} /(d-1) \text { for all } n .
$$

According to this theorem, if $\left\|\mathbf{v}^{n}\right\|_{\infty} \leqslant\left\|\mathbf{v}^{n}\right\|_{1} /\left(d_{\infty}-1\right)$ for all $n \geqslant n_{0}$, then Eq. (4) becomes $\Omega(\mathbf{v})=\Omega^{n_{0}}+\left\|\mathbf{v}^{n_{0}}\right\|_{1} /\left(d_{\infty}-1\right)$. In particular, if $n_{0}=1$, we get

$$
\Omega(\mathbf{v})=\frac{\|\mathbf{v}\|_{1}}{d-1}
$$

\section{Connectedness at the Connecting Thickness}

By definition of $\Omega(\mathbf{v}, \mu), \mathbb{P}(\mathbf{v}, \mu, \theta)$ is disconnected for all $\theta<\Omega(\mathbf{v}, \mu)$, and connected for all $\theta>\Omega(\mathbf{v}, \mu)$. The question we want to answer now is whether $\mathbb{P}(\mathbf{v}, \mu, \Omega(\mathbf{v}, \mu))$ is connected. This question has an easy answer when OFS terminates, which means when $\operatorname{dim}_{\mathbb{Q}}(\mathbf{v})=1$. 
Theorem 4. If $\operatorname{dim}_{\mathbb{Q}}(\mathbf{v})=1$ then $\mathbb{P}(\mathbf{v}, \mu, \Omega(\mathbf{v}, \mu))$ is disconnected for all $\mu \in \mathbb{R}$.

Proof. When $\operatorname{dim}_{\mathbb{Q}}(\mathbf{v})=1, \operatorname{gcd}\left(\mathbf{v}^{n}\right)$ is obviously an invariant of OFS. Therefore, when the halting condition is reached, we have $\mathbf{v}^{n}=\gamma \mathbf{e}_{1}$ where $\gamma=$ $\operatorname{gcd}\left(v_{1}, \ldots, v_{d}\right)$. Then $\mathbb{P}(\mathbf{v}, \mu, \Omega(\mathbf{v}, \mu))$ is connected if and only if $\mathbb{P}\left(\mathbf{v}^{n}, \mu, \Omega\left(\mathbf{v}^{n}, \mu\right)\right)$ is connected. But $\Omega\left(\mathbf{v}^{n}, \mu\right)=\mu \bmod \gamma$, and $\mathbb{P}\left(\mathbf{v}^{n}, \mu, \Omega\left(\mathbf{v}^{n}, \mu\right)\right)=\{x \in \mathbb{Z} \mid 0 \leqslant$ $\gamma x+\mu<\mu \bmod \gamma\}$. This set is empty, hence disconnected.

If OFS does not terminate, then $\Omega(\mathbf{v}, \mu)=\Omega(\mathbf{v})$, and after some step $n_{0}$, no coordinate of the vector vanishes anymore, meaning that $v_{1}^{n}>0$ for all $n \geqslant n_{0}$. Then $\mathbb{P}(\mathbf{v}, \mu, \Omega(\mathbf{v}))$ is connected if and only if $\mathbb{P}\left(\mathbf{v}^{n_{0}}, \mu, \Omega\left(\mathbf{v}^{n_{0}}\right)\right)$ is connected. Therefore, we shall now study the case of vectors such that $v_{1}^{n}>0$ for all $n$.

Theorem 5. If $v_{1}^{n}>0$ for all $n$, and $\left\|\mathbf{v}^{n}\right\|_{\infty} \geqslant\left\|\mathbf{v}^{n}\right\|_{1} /(d-1)$ for some $n$, then $\mathbb{P}(\mathbf{v}, \mu, \Omega(\mathbf{v}))$ is disconnected for all $\mu \in \mathbb{R}$.

The following lemma will be useful for the proof of this theorem.

Lemma 6. Let $d \geqslant 3$ and $\mathbf{v} \in \mathcal{O}_{d}^{+}$. If there exists $r \in\{2, \ldots, d-1\}$ such that $\operatorname{dim}_{\mathbb{Q}}\left(v_{1}, \ldots, v_{r}\right) \geqslant 2$, and $v_{r+1} \geqslant \Omega\left(v_{1}, \ldots, v_{r}\right)$ then $\Omega(\mathbf{v})=v_{d}$.

Proof. If $v_{1}=0$ then we have $r \geqslant 3$ because $\operatorname{dim}_{\mathbb{Q}}\left(v_{1}, \ldots, v_{r}\right) \geqslant 2$, and the conditions of the theorem still hold for $\left(v_{2}, \ldots, v_{d}\right)$, taking $d^{\prime}=d-1$ and $r^{\prime}=$ $r-1$. We may therefore assume, without loss of generality, that $v_{1}>0$. Then

$$
\Omega(\mathbf{v})=v_{1}+\Omega\left(v_{1}, v_{2}-v_{1}, \ldots, v_{r}-v_{1}, v_{r+1}-v_{1}, \ldots, v_{d}-v_{1}\right),
$$

and

$$
\Omega\left(v_{1}, \ldots, v_{r}\right)=v_{1}+\Omega\left(v_{1}, v_{2}-v_{1}, \ldots, v_{r}-v_{1}\right) .
$$

Let $\mathbf{v}^{\prime}=\left(v_{1}, v_{2}-v_{1}, \ldots, v_{d}-v_{1}\right)$ and $\mathbf{v}^{\prime \prime}=\operatorname{sort}\left(\mathbf{v}^{\prime}\right)$. We have $v_{r}^{\prime}>0$ since otherwise we would have $v_{1}=\cdots=v_{r}$, hence $\operatorname{dim}_{\mathbb{Q}}\left(v_{1}, \ldots, v_{r}\right)=1$. We also have $\operatorname{dim}_{\mathbb{Q}}\left(v_{1}^{\prime}, \ldots, v_{r}^{\prime}\right)=\operatorname{dim}_{\mathbb{Q}}\left(v_{1}, \ldots, v_{r}\right) \geqslant 2$. Hence, $\left(v_{1}^{\prime}, \ldots, v_{r}^{\prime}\right)$ has at least two non zero coordinates. By hypothesis, we have $v_{r+1}^{\prime}=v_{r+1}-v_{1} \geqslant$ $\Omega\left(v_{1}, v_{2}, \ldots, v_{r}\right)-v_{1}=\Omega\left(v_{1}, v_{2}-v_{1}, \ldots, v_{r}-v_{1}\right)=\Omega\left(v_{1}^{\prime}, v_{2}^{\prime}, \ldots, v_{r}^{\prime}\right)$, which, by Th. 1] implies $v_{r+1}^{\prime} \geqslant\left\|\left(v_{1}^{\prime}, \ldots, v_{r}^{\prime}\right)\right\|_{\infty}$. Hence, $\left(v_{1}^{\prime \prime}, \ldots, v_{r}^{\prime \prime}\right)=\operatorname{sort}\left(v_{1}^{\prime}, \ldots, v_{r}^{\prime}\right)$, and $\left(v_{r+1}^{\prime \prime}, \ldots, v_{d}^{\prime \prime}\right)=\left(v_{r+1}^{\prime}, \ldots, v_{d}^{\prime}\right)=\left(v_{r+1}-v_{1}, \ldots, v_{d}-v_{1}\right)$. Thus, $\mathbf{v}^{\prime \prime}$ still satisfies the conditions of the theorem. Furthermore, when we apply OFS to $\mathbf{v}$, for all $n$, we have $\left\|\mathbf{v}^{n}\right\|_{\infty}=v_{\# \mathbf{v}^{n}}^{n}=v_{d}-\Omega^{n}$. Therefore, $\Omega(\mathbf{v})=\Omega^{\infty}+\left\|\mathbf{v}^{\infty}\right\|_{\infty}=$ $\Omega^{\infty}+\left(v_{d}-\Omega^{\infty}\right)=v_{d}$.

Proof of Th. 5 (sketch). For all $n \geqslant 1$ and all $r \in\{2, \ldots, d\}$, we consider $K_{r}^{n}=$ $(r-1) v_{r}^{n}-\left(v_{1}^{n}+\cdots+v_{r}^{n}\right)$. We check that, as long as $v_{1}^{n}>0, K_{r}^{n}$ is always increasing, both in $r$ and in $n$. We define $r_{0}$ as the smallest index $r$ such that $K_{r}^{n} \geqslant 0$ for some $n$, and $n_{0}$ as the smallest index $n$ such that $K_{r_{0}}^{n} \geqslant 0$. Since $K_{2}^{n}=-v_{1}^{n}<0$, we have $r_{0} \geqslant 3$.

We prove that $\operatorname{dim}_{\mathbb{Q}}\left(v_{1}^{n_{0}}, \ldots, v_{r_{0}-1}^{n_{0}}\right) \geqslant 2$, and $\Omega\left(v_{1}^{n_{0}}, \ldots, v_{r_{0}-1}^{n_{0}}\right)=\left(v_{1}^{n_{0}}+\cdots+\right.$ $\left.v_{r_{0}-1}^{n_{0}}\right) /\left(r_{0}-2\right) \leqslant v_{r_{0}}^{n_{0}}$. By Lemma. 6. we get $\Omega\left(\mathbf{v}^{n_{0}}\right)=v_{d}^{n_{0}}=\left\|\mathbf{v}^{n_{0}}\right\|_{\infty}$. Then, by Th. 1. $\mathbb{P}\left(\mathbf{v}^{n_{0}}, \mu, \Omega\left(\mathbf{v}^{n_{0}}\right)\right)$, hence $\mathbb{P}(\mathbf{v}, \mu, \Omega(\mathbf{v}))$, is disconnected for all $\mu \in \mathbb{R}$. 
We are now left to consider the case where $v_{1}^{n}>0$ and $\left\|\mathbf{v}^{n}\right\|_{1}>(d-1)\left\|\mathbf{v}^{n}\right\|_{\infty}$ for all $n$. That is to say, where $v_{1}^{n}>0$ for all $n$ but $\mathbf{v}$ does not satisfy the second condition of Th. 5. For $d \geqslant 2$, We note $\mathcal{K}_{d}$ the set of such vectors. Kraaikamp \& Meester [9] have shown that $\mathcal{K}_{d}$ is Lebesgue negligible for all $d \geqslant 3$. If $\mathbf{v} \in \mathcal{K}_{d}$ then, by Th. 3. $\mathbf{v}^{\infty}=0$ so that $\Omega(\mathbf{v})=\|\mathbf{v}\|_{1} /(d-1)$. From what precedes, it is the only case where $v_{1}^{n}>0$ for all $n$, and $\mathbb{P}(\mathbf{v}, \mu, \Omega(\mathbf{v}))$ could possibly be connected. Some partial results have already been published in the literature.

A first result was obtained in 2 for the case where $\mathbf{v}=\left(\alpha, \alpha+\alpha^{2}, 1\right)$, and $\alpha$ is the inverse of the Tribonacci number, which means $\alpha^{3}+\alpha^{2}+\alpha-1=0$. We have $\mathbf{v} \in \mathcal{K}_{3}$, and it has been shown that $\mathbb{P}(\mathbf{v}, 0, \Omega(\mathbf{v}))$ is connected, and $\mathbb{P}(\mathbf{v}, \Omega(\mathbf{v}), \Omega(\mathbf{v}))$ is disconnected.

For the case $d=3$, it has been shown in 3 that $\mathbb{P}(\mathbf{v}, 0, \Omega(\mathbf{v}))$ is connected for all vectors in $\mathcal{K}_{3}$. The proof relies on techniques from the field of substitutions on planes, and seems difficult to extend to higher dimensions.

In the sequel, we shall address the general case, and prove that $\mathbb{P}(\mathbf{v}, 0, \Omega(\mathbf{v}))$ is connected for all $\mathbf{v} \in \cup_{d \geqslant 2} \mathcal{K}_{d}$. Note that in the case $d=2$, the condition of Kraaikamp \& Meester becomes $v_{2}^{n}<v_{1}^{n}+v_{2}^{n}$ which always holds if $v_{1}^{n}>0$ for all $n$, i.e. if $\operatorname{dim}_{\mathbb{Q}}\left(v_{1}, v_{2}\right)=2$. In this case, we have $\Omega\left(v_{1}, v_{2}\right)=v_{1}+v_{2}=\|\mathbf{v}\|_{1}$. By Th. 1] $\mathbb{P}(\mathbf{v}, \mu, \Omega(\mathbf{v}))$ is connected for all $\mu \in \mathbb{R}$.

\section{Main Connectedness Result}

In order to establish the main result of this work, we need to study carefully the behaviour of the fully subtractive algorithm. To do so, we consider an unordered version of this algorithm, which means that the coordinates of the vector are not ordered anymore. This works as follows: as long as $\# \mathbf{v} \geqslant 2$, if some coordinate is zero, it is erased, otherwise, a minimal coordinate is subtracted from all other ones. We call this algorithm UFS for Unordered Fully Subtractive.

For all $k \geqslant 1$, we define $\sigma_{k}$ and $\pi_{k}$ as:

$$
\begin{aligned}
\sigma_{k}: \bigcup_{d \geqslant k} \mathbb{R}^{d} & \rightarrow \bigcup_{d \geqslant k} \mathbb{R}^{d} \\
\left(v_{1}, \ldots, v_{k}, \ldots, v_{d}\right) & \mapsto\left(v_{1}-v_{k}, \ldots, v_{k-1}-v_{k}, v_{k}, v_{k+1}-v_{k}, \ldots, v_{d}-v_{k}\right) ; \\
\pi_{k}: \bigcup_{d \geqslant k} \mathbb{R}^{d} & \rightarrow \bigcup_{d \geqslant k} \mathbb{R}^{d-1} \\
\left(v_{1}, \ldots, v_{k}, \ldots, v_{d}\right) & \mapsto\left(v_{1}, \ldots, v_{k-1}, v_{k+1}, \ldots, v_{d}\right) .
\end{aligned}
$$

UFS computes a possibly infinite sequence of pairs $\left(\mathbf{v}^{n}, \Omega^{n}\right)_{n \geqslant 1}$ defined by:

$$
\begin{aligned}
& \left(\mathbf{v}^{1}, \Omega^{1}\right)=(\mathbf{v}, 0), \text { and for all } n \geqslant 1 \text { such that } \# \mathbf{v}^{n} \geqslant 2, \\
& \left(\mathbf{v}^{n+1}, \Omega^{n+1}\right)= \begin{cases}\left(\pi_{i_{0}}\left(\mathbf{v}^{n}\right), \Omega^{n}\right) & \text { if } v_{i_{0}}^{n}=0 \\
\left(\sigma_{i_{0}}\left(\mathbf{v}^{n}\right), \Omega^{n}+v_{i_{0}}^{n}\right) & \text { if } v_{i_{0}}^{n}=\min _{i} v_{i}^{n}>0 .\end{cases}
\end{aligned}
$$

Note that UFS is not deterministic since several coordinates of $\mathbf{v}^{n}$ could be minimal. However, OFS and UFS generate the same sequence $\left(\Omega^{n}\right)_{n \geqslant 1}$ and if 
$\left(\mathbf{v}^{n}\right)_{n \geqslant 1}$ and $\left(\mathbf{v}^{\prime n}\right)_{n \geqslant 1}$ are the sequences of vectors generated respectively by OFS and UFS, we have $\mathbf{v}^{n}=\operatorname{sort}\left(\mathbf{v}^{\prime n}\right)$ for all $n$.

From now on, we consider a vector $\mathbf{v}$ in $\left(\mathbb{R}^{+}\right)^{d}$ such that UFS never erases a coordinate, meaning that $\min _{i} v_{i}^{n}>0$ for all $n$. We consider the infinite sequence $\Delta(\mathbf{v})=\left(\delta_{n}\right)_{n \geqslant 1} \in\{1, \ldots, d\}^{\omega}$ where $\delta_{n}$ is the index of the minimal coordinate of $\mathbf{v}^{n}$ which is subtracted from all other ones. For all $n \geqslant 1$, we have $\Delta(\mathbf{v})=$ $\delta_{1} \cdots \delta_{n_{0}-1} \Delta\left(\mathbf{v}^{n}\right), \mathbf{v}^{n}=\sigma_{\delta_{n-1}} \ldots \sigma_{\delta_{1}}(\mathbf{v})$, and $\Omega^{n}=v_{\delta_{1}}^{1}+\cdots+v_{\delta_{n-1}}^{n-1}$. We set $\theta_{n}=v_{\delta_{n}}^{n}$ so that $\Omega^{n}=\sum_{i=1}^{n-1} \theta_{i}$. Note that, by hypothesis, $\theta_{n}>0$ for all $n$. We have

$$
\theta_{n}=v_{\delta_{n}}^{n}=\left\langle\mathbf{v}^{n}, \mathbf{e}_{\delta_{n}}\right\rangle=\left\langle\sigma_{\delta_{n-1}} \ldots \sigma_{\delta_{1}}(\mathbf{v}), \mathbf{e}_{\delta_{n}}\right\rangle=\left\langle\mathbf{v},{ }^{t} \sigma_{\delta_{1}} \ldots{ }^{t} \sigma_{\delta_{n-1}}\left(\mathbf{e}_{\delta_{n}}\right)\right\rangle
$$

where ${ }^{t} \sigma$ denotes the transpose of $\sigma$. We set $\mathbf{T}_{n}={ }^{t} \sigma_{\delta_{1}} \ldots{ }^{t} \sigma_{\delta_{n-1}}\left(\mathbf{e}_{\delta_{n}}\right)$ so that, for all $n$, we have $\left\langle\mathbf{v}, \mathbf{T}_{n}\right\rangle=\theta_{n}$. The sequence $\left(\mathbf{T}_{n}\right)_{n \geqslant 1}$ has some nice properties.

Lemma 7 ([7]). If the first occurrence in $\Delta(\mathbf{v})$ of some $k \in\{1, \ldots, d\}$ is at position $n$, meaning that $\delta_{n}=k$, and $\delta_{i} \neq k$ for all $i<n$, then $\mathbf{T}_{1}+\cdots+\mathbf{T}_{n}=\mathbf{e}_{k}$.

Lemma 8 ([7]). If $m$ and $n$ are the positions of two consecutive occurrences in $\Delta(\mathbf{v})$ of some $k \in\{1, \ldots, d\}$, meaning that $m<n, \delta_{m}=\delta_{n}=k$ and $\delta_{i} \neq k$ for all $i \in\{m+1, \ldots, n-1\}$, then $\mathbf{T}_{m+1}+\cdots+\mathbf{T}_{n}=\mathbf{T}_{m}$.

Lemma 9 ([]]). If $i \leqslant j<k$ and $\delta_{j} \neq \delta_{k}$ then $\left\langle\mathbf{v},\left(\mathbf{T}_{1}+\cdots+\mathbf{T}_{k}\right)+\mathbf{T}_{i}\right\rangle \geqslant \Omega^{\infty}$. Therefore, $\left(\mathbf{T}_{1}+\cdots+\mathbf{T}_{k}\right)+\mathbf{T}_{i} \notin \mathbb{P}(\mathbf{v}, 0, \Omega(\mathbf{v}))$.

We recall now the construction of the geometric palindromic closure of $\Delta(\mathbf{v})$ 7. This construction builds incrementally a connected subset of $\mathbb{Z}^{d}$, which is easily shown to be included in $\mathbb{P}(\mathbf{v}, 0, \Omega(\mathbf{v}))$. We shall show that it in fact exactly $\mathbb{P}(\mathbf{v}, 0, \Omega(\mathbf{v}))$ when $\mathbf{v} \in \mathcal{K}_{d}$.

We define a sequence $\left(\mathbb{P}_{n}\right)_{n \geqslant 0}$ of subsets of $\mathbb{Z}^{d}$ by:

$$
\mathbb{P}_{0}=\{\mathbf{0}\} \text {, and } \mathbb{P}_{n}=\mathbb{P}_{n-1} \cup\left(\mathbb{P}_{n-1}+\mathbf{T}_{n}\right) \text { for } n \geqslant 1 \text {. }
$$

Theorem $10([\mathbf{7}]) . \mathbb{P}_{\infty}=\lim _{n \rightarrow \infty} \mathbb{P}_{n}$ is connected.

The set $\mathbb{P}_{\infty}$ is the geometric palindromic closure of $\Delta(\mathbf{v})$ in $\mathbb{Z}^{d}[7$. From the definition of $\mathbb{P}_{n}$, we get the following characterisation:

$$
\begin{aligned}
& \mathbb{P}_{n}=\left\{\sum_{i \in I} \mathbf{T}_{i} \mid I \subseteq\{1, \ldots, n\}\right\} \quad \text { for all } n \geqslant 0 ; \\
& \mathbb{P}_{\infty}=\left\{\sum_{i \in I} \mathbf{T}_{i}\left|I \subset \mathbb{N}_{1},\right| I \mid<\infty\right\} .
\end{aligned}
$$

The inclusion $\mathbb{P}_{\infty} \subseteq \mathbb{P}(\mathbf{v}, 0, \Omega(\mathbf{v}))$ is straightforward. From what precedes, each $\mathbf{x}$ in $\mathbb{P}_{\infty}$ may be written as $\mathbf{x}=\sum_{i \in I} \mathbf{T}_{i}$, for some finite subset $I$ of $\mathbb{N}_{1}$. Then $\langle\mathbf{v}, \mathbf{x}\rangle=\sum_{i \in I}\left\langle\mathbf{v}, \mathbf{T}_{i}\right\rangle=\sum_{i \in I} \theta_{i} \in[0 ; \Omega(\mathbf{v})[$. Hence, $\mathbf{x}$ belongs to $\mathbb{P}(\mathbf{v}, 0, \Omega(\mathbf{v}))$. In the sequel, we prove that we have also $\mathbb{P}(\mathbf{v}, 0, \Omega(\mathbf{v})) \subseteq \mathbb{P}_{\infty}$, provided that each $k \in\{1, \ldots, d\}$ occurs infinitely many times in $\Delta(\mathbf{v})$. The lemma below states that it is the case if $\mathbf{v} \in \mathcal{K}_{d}$. 
Lemma 11. If $\mathbf{v} \in \mathcal{K}_{d}$ then each $k \in\{1, \ldots, d\}$ occurs infinitely many times in $\Delta(\mathbf{v})$.

Proof. Assume, by contradiction, that $\mathbf{v} \in \mathcal{K}_{d}$, and some $k \in\{1, \ldots, d\}$ does not occur anymore in $\Delta(\mathbf{v})$ for $n \geqslant n_{0}$. Since $\mathbf{v} \in \mathcal{K}_{d} \Longleftrightarrow \mathbf{v}^{n_{0}} \in \mathcal{K}_{d}$, and $\Delta(\mathbf{v})=\delta_{1} \cdots \delta_{n_{0}-1} \Delta\left(\mathbf{v}^{n_{0}}\right)$, we may assume, without loss of generality, that $n_{0}=1$, i.e. that $k$ never occurs in $\Delta(\mathbf{v})$. Then for all $n>1$, we have $v_{k}^{n}=$ $v_{k}^{n-1}-\theta_{n-1}=v_{k}-\sum_{i=1}^{n-1} \theta_{i}=v_{k}-\Omega^{n}$. Since $\mathbf{v} \in \mathcal{K}_{d}$, we have $\lim _{n \rightarrow \infty} \mathbf{v}^{n}=\mathbf{0}$, so that $v_{k}=\lim _{n \rightarrow \infty} \Omega^{n}=\Omega^{\infty}=\|\mathbf{v}\|_{1} /(d-1)$. But $v_{k} \leqslant\|\mathbf{v}\|_{\infty}$ and, by assumption, $\|\mathbf{v}\|_{\infty}<\|\mathbf{v}\|_{1} /(d-1)$. Hence a contradiction.

From now on, we assume that $\mathbf{v}$ belongs to $\mathcal{K}_{d}$, so that each $k \in\{1, \ldots, d\}$ occurs infinitely many times in $\Delta(\mathbf{v})$. To prove our main theorem, we still need some additional technical results. The first lemma below is an immediate consequence of the proof of Th. 13 in [7].

Lemma $12([\mathbf{7}])$. For all $\mathbf{x}, \mathbf{y}$ in $\mathbb{P}_{\infty}$, we have $\mathbf{x}-\mathbf{y} \in \mathbb{P}_{\infty}$ or $\mathbf{y}-\mathbf{x} \in \mathbb{P}_{\infty}$.

Theorem 13. Each $\mathbf{x}$ in $\mathbb{Z}^{d}$ may be written as $\pm\left(\alpha_{1} \mathbf{T}_{1}+\cdots+\alpha_{m} \mathbf{T}_{m}\right)$ for some $m \geqslant 0$ and $\alpha_{1}, \ldots, \alpha_{m} \in \mathbb{N}_{0}$.

Proof. Let $\mathbf{x} \in \mathbb{Z}^{d}$. We have $\mathbf{x}=x_{1} \mathbf{e}_{1}+\cdots+x_{d} \mathbf{e}_{d}$. From lemma [7, each $\mathbf{e}_{k}$ may be written as $\sum_{j=1}^{n_{k}} \mathbf{T}_{j}$ where $n_{k}$ is the index of the first occurrence of $k$ in $\Delta(\mathbf{v})$. Then $\mathbf{x}=\sum_{j=1}^{r} y_{j} \mathbf{T}_{j}$ for some $r \geqslant 0$ and $y_{1}, \ldots, y_{r} \in \mathbb{Z}$. Since $\mathbf{T}_{i}$ 's belong to $\mathbb{P}_{\infty}$, this last sum may always be decomposed as $\left(\mathbf{U}_{1}+\cdots+\mathbf{U}_{p}\right)-\left(\mathbf{V}_{1}+\cdots+\mathbf{V}_{q}\right)$ for some $p, q \geqslant 0$ where $\mathbf{U}_{i}$ 's and $\mathbf{V}_{i}$ 's belong to $\mathbb{P}_{\infty}$. If $p>0$ and $q>0$ then either $\mathbf{U}_{1}-\mathbf{V}_{1}=0$, in which case we may simply remove $\mathbf{U}_{1}$ and $\mathbf{V}_{1}$ from the sum, or by lemma [12, we have either $\mathbf{U}_{1}-\mathbf{V}_{1}=\mathbf{U}^{\prime}{ }_{1}$ where $\mathbf{U}^{\prime}{ }_{1} \in \mathbb{P}_{\infty}$ or $\mathbf{U}_{1}-\mathbf{V}_{1}=-\mathbf{V}^{\prime}{ }_{1}$ where $\mathbf{V}^{\prime}{ }_{1} \in \mathbb{P}_{\infty}$. Replacing $\mathbf{U}_{1}-\mathbf{V}_{1}$ with either $\mathbf{U}^{\prime}{ }_{1}$ or $-\mathbf{V}^{\prime}{ }_{1}$, the number of terms in the sum decreases. Repeating this process as long as $p>0$ and $q>0$, yields an expression of $\mathbf{x}$ as $\pm\left(\mathbf{W}_{1}+\cdots+\mathbf{W}_{s}\right)$ for some $s \geqslant 0$ and $\mathbf{W}_{1}, \ldots, \mathbf{W}_{s} \in \mathbb{P}_{\infty}$. Now, each $\mathbf{W}_{i}$ may be written as a finite sum of $\mathbf{T}_{j}$ 's. Doing so, and collecting the $\mathbf{T}_{j}$ 's yields the result.

Corollary 14. Each $\mathbf{x}$ in $\mathbb{P}(\mathbf{v}, 0, \Omega(\mathbf{v}))$ may be written as $\alpha_{1} \mathbf{T}_{1}+\cdots+\alpha_{m} \mathbf{T}_{m}$ for some $m \geqslant 0$ and $\alpha_{1}, \ldots, \alpha_{m} \in \mathbb{N}_{0}$.

Proof. We have $\mathbf{x}=\varepsilon \times\left(\alpha_{1} \mathbf{T}_{1}+\cdots+\alpha_{m} \mathbf{T}_{m}\right)$ for some $\varepsilon= \pm 1, m \geqslant 0$, and $\alpha_{1}, \ldots, \alpha_{m} \in \mathbb{N}_{0}$. Then $\langle\mathbf{v}, \mathbf{x}\rangle=\varepsilon \times\left(\alpha_{1}\left\langle\mathbf{v}, \mathbf{T}_{1}\right\rangle+\cdots+\alpha_{m}\left\langle\mathbf{v}, \mathbf{T}_{m}\right\rangle\right)=$ $\varepsilon \times\left(\alpha_{1} \theta_{1}+\cdots+\alpha_{m} \theta_{m}\right)$. Since $\mathbf{x} \in \mathbb{P}(\mathbf{v}, 0, \Omega(\mathbf{v}))$ we have $\langle\mathbf{v}, \mathbf{x}\rangle \geqslant 0$, so that $\varepsilon=+1$ because $\theta_{i}>0$ for all $i$.

Given a nonempty subset $X$ of $\mathbb{Z}$, we denote by $X^{\star} 0^{\omega}$ the set of infinite sequences of which the terms belong to $X$, and containing only finitely many non-zero terms. We define a linear mapping $\psi$ from the $\mathbb{Z}$-module $\mathbb{Z}^{\star} 0^{\omega}$ to $\mathbb{Z}^{d}$ by $\psi(W)=\sum_{i \geqslant 1} w_{i} \mathbf{T}_{i}$. Then $\mathbb{P}_{\infty}=\psi\left(\{0,1\}^{\star} 0^{\omega}\right)=\left\{\psi(W) \mid W \in\{0,1\}^{\star} 0^{\omega}\right\}$. Next lemma is the immediate reformulation of Lemmas 7 and 8 in terms of $\psi$. 


\section{Lemma 15}

- If $n$ is the index of the first occurrence of $k$ in $\Delta(\mathbf{v})$, then $\psi\left(1^{n} 0^{\omega}\right)=e_{k}$.

- If $m$ and $n$ are the indexes of two consecutive occurrences of $k$ in $\Delta(\mathbf{v})$, then $\psi\left(0^{m-1} 01^{n-m} 0^{\omega}\right)=\psi\left(0^{m-1} 10^{n-m} 0^{\omega}\right)$.

To prove that $\mathbb{P}(\mathbf{v}, 0, \Omega(\mathbf{v}))=\mathbb{P}_{\infty}$, it is sufficient to prove that each $\mathbf{x}$ in $\mathbb{P}(\mathbf{v}, 0, \Omega(\mathbf{v}))$ may be written as $\psi(W)$ for some $W$ in $\{0,1\}^{\star} 0^{\omega}$. Thanks to Cor. 14, we may find $W \in \mathbb{N}_{0}^{\star} 0^{\omega}$ such that $\mathbf{x}=\psi(W)$. Using Lemma 15, we shall transform $W$ into $W^{\prime} \in\{0,1\}^{\star} 0^{\omega}$ such that $\psi\left(W^{\prime}\right)=\psi(W)$. We define two transformations on $\mathbb{N}_{0}^{\star} 0^{\omega}$ as follows.

$$
\begin{aligned}
& \begin{array}{rlrllll}
i & = & & m & & & \\
& & & & \\
\Delta & = & k & \ldots \ldots \ldots & k & \ldots
\end{array} \\
& W=\cdots \quad 0 \quad w_{m+1}^{\prime}+1 \cdots w_{n}^{\prime}+1 \cdots \\
& \rightarrow W^{\prime}=\cdots \quad 1 \quad w_{m+1}^{\prime} \quad \cdots \quad w_{n}^{\prime} \quad \cdots
\end{aligned}
$$

\section{Reduction}

\section{Expansion}

$$
\begin{aligned}
& W=\cdots u+2 \quad w_{m+1} \quad \cdots w_{n} \quad \cdots \\
& \rightarrow W^{\prime}=\cdots u+1 \quad w_{m+1}+1 \cdots w_{n}+1 \cdots
\end{aligned}
$$

where $m<n$, and $u, w_{m+1}, \ldots, w_{n}, w_{m+1}^{\prime}, \ldots, w_{n}^{\prime} \geqslant 0$, and $\delta_{i} \neq k$ for all $i \in$ $\{m+1, \ldots, n-1\}$.

Since $\psi$ is linear, according to Lemma 15, both these transformations preserve $\psi(W)$. Given $W \in \mathbb{N}_{0}^{\star} 0^{\omega}$, we apply these two transformations with the following strategy.

First apply Reduction as much as possible.

Then, as long as $w_{i} \geqslant 2$ for some $i$ :

1. apply Expansion once at the last position $m$ such that $w_{m} \geqslant 2$;

2. apply Reduction as much as possible.

This strategy, if it terminates, obviously yields a sequence in $\{0,1\}^{\star} 0^{\omega}$ since otherwise Expansion would still apply.

Theorem 16. If $W \in \mathbb{N}_{0}^{\star} 0^{\omega}$ and $\psi(W) \in \mathbb{P}(\mathbf{v}, 0, \Omega(\mathbf{v}))$, then applying Reduction and Expansion to $W$ with the strategy above, terminates and yields $W^{\prime} \in\{0,1\}^{\star} 0^{\omega}$ such that $\psi\left(W^{\prime}\right)=\psi(W)$.

Before proving this theorem, let us introduce some more notation. Given a sequence $W$ in $\mathbb{N}_{0}^{\star},|W|$ is the length of $W$. If $W \in \mathbb{N}_{0}^{\star} 0^{\omega}$ then $|W|$ is the index of the last non-zero term in $W$, or 0 if $W=0^{\omega}$.

Proof of Th.16 (sketch). We consider the multiset $M$ of all terms in $W$ which are greater than 2. Our strategy ensures that $M$ never increases. It decreases each time an expansion is performed on $w_{m} \cdots w_{n}$ if $w_{m} \geqslant 3$. It also decreases 
when a reduction is performed on $w_{m} \cdots w_{n}$ if a position $i$ exists between $m+1$ and $n$ such that $w_{i} \geqslant 3$. Hence, after finitely many transformation steps, for each transformation, we have $w_{i} \leqslant 2$ for all $i \in[m ; n]$, and $M$ does not evolve anymore. It is therefore sufficient to prove termination when $W \in\{0,1,2\}^{\star} 0^{\omega}$.

We observe that each transformation decreases $W$ in the lexicographic ordering induced by $1<2<0$, so that the transformation process never loops. Although this ordering is not a well-order on infinite sequences, it is on sequences with bounded length. Each transformation which does not increase $|W|$, may be seen as operating on $\{0,1,2\}^{|W|}$. Therefore, there may be only finitely many reductions between two expansions, and the transformation process may not terminate only if it performs infinitely many expansions which increase $|W|$.

Finally, let $\rho_{2}(W)$ be the number of maximal sub-sequences in $W$ containing no 0 , and containing at least one 2 . A careful examination of the transformation rules shows that $\rho_{2}(W)$ never increases. A case analysis shows that each expansion step which increases $|W|$ is eventually followed by a reduction step which reduces $\rho_{2}(W)$. The transformation process therefore terminates.

From this theorem and Cor. 14, we deduce that each $\mathbf{x}$ in $\mathbb{P}(\mathbf{v}, 0, \Omega(\mathbf{v}))$ may be written as $\psi(W)$ with $W \in\{0,1\}^{\star} 0^{\omega}$, and therefore $\mathbb{P}(\mathbf{v}, 0, \Omega(\mathbf{v})) \subseteq \mathbb{P}_{\infty}$.

Theorem 17. Let $d \geqslant 2$. For all $\mathbf{v}$ in $\mathcal{K}_{d}, \mathbb{P}(\mathbf{v}, 0, \Omega(\mathbf{v}))$ is connected.

As a corollary we get the following result.

Corollary 18. For all $\mathbf{v} \in \mathcal{K}_{d}$, we have $\operatorname{dim}_{\mathbb{Q}}(\mathbf{v})=d$.

Proof. Assume that $\operatorname{dim}_{\mathbb{Q}}(\mathbf{v})<d$. Then there exists $\mathbf{p} \neq \mathbf{0}$ in $\mathbb{Z}^{d}$ such that $\langle\mathbf{v}, \mathbf{p}\rangle=0$. Thus, $\mathbf{p} \in \mathbb{P}(\mathbf{v}, 0, \Omega(\mathbf{v}))$, hence $\mathbf{p}=\psi(U)$ for some $U \in\{0,1\}^{\star} 0^{\omega}$. Since $\mathbf{p} \neq \mathbf{0}$, we have $U \neq 0^{\omega}$. Then $\langle\mathbf{v}, \mathbf{p}\rangle=\sum_{i \geqslant 1} u_{i}\left\langle\mathbf{v}, \mathbf{T}_{i}\right\rangle=\sum_{i \geqslant 1} u_{i} \theta_{i}>0$. Hence a contradiction.

It should be noted that Kraaikamp \& Meester actually proved Th. 3 with the assumption that $\operatorname{dim}_{\mathbb{Q}}(\mathbf{v})=d$. However, they used this hypothesis only to ensure that $v_{1}^{n}>0$ for all $n$. As a matter of fact, an earlier version of this theorem exists [10] with only the assumption that $v_{1}^{n}>0$ for all $n$. With this weaker assumption, we get $\operatorname{dim}_{\mathbb{Q}}(\mathbf{v})=d$ as a corollary.

\section{Connectedness of Hyperplanes with Non-zero Shift}

We have established that for each $d \geqslant 2, \mathbb{P}(\mathbf{v}, 0, \Omega(\mathbf{v}))$ is connected for all $\mathbf{v} \in \mathcal{K}_{d}$. The question which arises naturally is whether $\mathbb{P}(\mathbf{v}, \mu, \Omega(\mathbf{v}))$ is still connected when $\mu \neq 0$. We already know that $\mathbb{P}(\mathbf{v}, \mu, \Omega(\mathbf{v}))$ is connected for all $\mu \in \mathbb{R}$ if $\mathbf{v} \in \mathcal{K}_{2}$. For $d \geqslant 3$, we don't have a general result. However, it has been established in [2], for a specific vector in $\mathcal{K}_{3}$, that $\mathbb{P}(\mathbf{v}, \mu, \Omega(\mathbf{v}))$ is disconnected if $\mu=\Omega(\mathbf{v})$. Theorem 20 below shows that this holds for all $\mathbf{v} \in \mathcal{K}_{d}$, for all $d \geqslant 3$.

Lemma $19([7])$. Let $\Delta \in\{1, \ldots, d\}^{\omega}$ and $\mathbb{P}_{\infty}$ be the geometric palindromic closure of $\Delta$. Then $\mathbb{P}_{\infty} \backslash\{\mathbf{0}\}$ has exactly as many connected components as the cardinal of $\left\{\delta_{i} \mid i \in \mathbb{N}_{1}\right\}$. 
If $\mathbf{v} \in \mathcal{K}_{d}$ then each $k \in\{1, \ldots, d\}$ occurs in $\Delta(\mathbf{v})$. Thus, $\mathbb{P}_{\infty} \backslash\{\mathbf{0}\}$ has exactly $d$ connected components, and is therefore disconnected since $d \geqslant 2$.

Theorem 20. If $d \geqslant 3$ and $\mathbf{v} \in \mathcal{K}_{d}$ then $\mathbb{P}(\mathbf{v}, \Omega(\mathbf{v}), \Omega(\mathbf{v}))$ is disconnected.

Proof. We have

$$
\begin{aligned}
& \mathbb{P}(\mathbf{v}, \Omega(\mathbf{v}), \Omega(\mathbf{v})) \\
& =\left\{\mathbf{x} \in \mathbb{Z}^{d} \mid 0 \leqslant\langle\mathbf{v}, \mathbf{x}\rangle+\Omega(\mathbf{v})<\Omega(\mathbf{v})\right\} \\
& =\left\{\mathbf{x} \in \mathbb{Z}^{d} \mid-\Omega(\mathbf{v}) \leqslant\langle\mathbf{v}, \mathbf{x}\rangle<0\right\} \\
& =-\left\{\mathbf{x} \in \mathbb{Z}^{d} \mid 0<\langle\mathbf{v}, \mathbf{x}\rangle \leqslant \Omega(\mathbf{v})\right\} \\
& =-\left(\left(\mathbb{P}(\mathbf{v}, \Omega(\mathbf{v}), \Omega(\mathbf{v})) \backslash\left\{\mathbf{x} \in \mathbb{Z}^{d} \mid\langle\mathbf{v}, \mathbf{x}\rangle=0\right\}\right) \cup\left\{\mathbf{x} \in \mathbb{Z}^{d} \mid\langle\mathbf{v}, \mathbf{x}\rangle=\Omega(\mathbf{v})\right\}\right) .
\end{aligned}
$$

Since $\mathbf{v} \in \mathcal{K}_{d}$, we have $\Omega(\mathbf{v})=\left(v_{1}+\cdots+v_{d}\right) /(d-1)$, and by Cor. [18, $\operatorname{dim}_{\mathbb{Q}}(\mathbf{v})=d$. Hence the only solution in $\mathbb{Q}^{d}$ of $\langle\mathbf{v}, \mathbf{x}\rangle=0$ is $\mathbf{x}=\mathbf{0}$, and the only solution of $\langle\mathbf{v}, \mathbf{x}\rangle=\Omega(\mathbf{v})$ is $x_{1}=\cdots=x_{d}=\frac{1}{d-1}$. Therefore, if $d \geqslant 3$, the equation $\langle\mathbf{v}, \mathbf{x}\rangle=$ $\Omega(\mathbf{v})$ has no solution in $\mathbb{Z}^{d}$. Hence, $\mathbb{P}(\mathbf{v}, \Omega(\mathbf{v}), \Omega(\mathbf{v}))=-\mathbb{P}(\mathbf{v}, 0, \Omega(\mathbf{v})) \backslash\{\mathbf{0}\}$, which, by Lemma 19, is disconnected.

\section{Summary of Results and Perspectives}

Let us now summarise the results of the previous sections about the connectedness of $\mathbb{P}(\mathbf{v}, \mu, \Omega(\mathbf{v}, \mu))$.

Theorem 21. Given $d \geqslant 2$ and $\mathbf{v} \in\left(\mathbb{R}^{+}\right)^{d} \backslash\{0\}$, we have the following results.

- If $\operatorname{dim}_{\mathbb{Q}}(\mathbf{v})=1$ then $\mathbb{P}(\mathbf{v}, \mu, \Omega(\mathbf{v}, \mu))$ is disconnected for all $\mu \in \mathbb{R}$.

- If $\operatorname{dim}_{\mathbb{Q}}(\mathbf{v}) \geqslant 2$ then let $\left(\mathbf{v}^{n}\right)_{n \geqslant 1}$ be the sequence of vectors computed by the fully subtractive algorithm applied to $\mathbf{v}$. We have $\Omega(\mathbf{v}, \mu)=\Omega(\mathbf{v})$ for all $\mu \in \mathbb{R}$, and $\mathbb{P}(\mathbf{v}, 0, \Omega(\mathbf{v}))$ is connected if and only if $\mathbf{v}^{n} \in \mathcal{K}_{d^{\prime}}$ for some $n$, and some $d^{\prime} \leqslant d$. In this case:

- If $d^{\prime}=2$ then $\mathbb{P}(\mathbf{v}, \mu, \Omega(\mathbf{v}))$ is connected for all $\mu \in \mathbb{R}$.

- If $d^{\prime} \geqslant 3$ then $\mathbb{P}(\mathbf{v}, \mu, \Omega(\mathbf{v}))$ is disconnected for some values of $\mu$. In particular, $\mathbb{P}(\mathbf{v}, \Omega(\mathbf{v}), \Omega(\mathbf{v}))$ is disconnected.

This theorem provides a complete characterisation of the connectedness of $\mathbb{P}(\mathbf{v}, 0, \theta)$ at the critical thickness $\theta=\Omega(\mathbf{v}, 0)$. For the case $d=3$, Th. 5.1 in 3 already established the connectedness of $\mathbb{P}(\mathbf{v}, 0, \Omega(\mathbf{v}, 0))$ for all $\mathbf{v} \in \mathcal{K}_{3}$. However, the only if part of that theorem is wrong. The authors claim falsely that the vectors in $\mathcal{K}_{3}$ are the only ones in $\left(\mathbb{R}^{+}\right)^{3}$ for which $\mathbb{P}(\mathbf{v}, 0, \Omega(\mathbf{v}))$ is connected. This is obviously false since $\mathbb{P}(\mathbf{v}, 0, \Omega(\mathbf{v}))$ is connected for all vectors of the form $\left(0, v_{2}, v_{3}\right)$ such that $\operatorname{dim}_{\mathbb{Q}}\left(v_{2}, v_{3}\right)=2$.

For $\mathbf{v} \in \mathcal{O}_{3}^{+} \backslash \mathcal{K}_{3}$, if $\operatorname{dim}_{\mathbb{Q}}(\mathbf{v})>1$, we have eventually $v_{3}^{n} \geqslant v_{1}^{n}+v_{2}^{n}$ for some $n$. The mistake in the proof lies in the argument that this implies $\Omega\left(\mathbf{v}^{n}\right)=v_{3}^{n}=$ $\left\|\mathbf{v}^{n}\right\|_{\infty}$. Then, by Th. 1, $\mathbb{P}\left(\mathbf{v}^{n}, 0, \Omega\left(\mathbf{v}^{n}\right)\right)$ would be disconnected. Actually, we have $\Omega\left(\mathbf{v}^{n}\right)=v_{3}^{n}$ only if $\operatorname{dim}_{\mathbb{Q}}\left(v_{1}^{n}, v_{2}^{n}\right)=2$. When $\operatorname{dim}_{\mathbb{Q}}\left(v_{1}^{n}, v_{2}^{n}\right)=1$, we have eventually $v_{1}^{m}=0$ for some $m$, and then $\mathbf{v}^{m+1}=\left(v_{2}^{m}, v_{3}^{m}\right)$. Since $\operatorname{dim}_{\mathbb{Q}}\left(\mathbf{v}^{m+1}\right)=$ 
$\operatorname{dim}_{\mathbb{Q}}(\mathbf{v})>1$, we have $\mathbf{v}^{m+1} \in \mathcal{K}_{2}$. Then $\mathbb{P}\left(\mathbf{v}^{m+1}, 0, \Omega\left(\mathbf{v}^{m+1}\right)\right)$ is connected, and $\mathbb{P}(\mathbf{v}, 0, \Omega(\mathbf{v}))$ as well. In this case, $\mathbb{P}(\mathbf{v}, \mu, \Omega(\mathbf{v}))$ is actually connected for all $\mu \in \mathbb{R}$. As a matter of fact, we have $\Omega\left(\mathbf{v}^{n}\right)=v_{3}^{n}+\operatorname{gcd}\left(v_{1}^{n}, v_{2}^{n}\right)$. Take for instance $\mathbf{v}=(1,1, \sqrt{2}+1)$. This vector does not belong to $\mathcal{K}_{3}$, but after one application of OFS, we get $\mathbf{v}^{2}=(0,1, \sqrt{2})$ which reduces to $\mathbf{v}^{3}=(1, \sqrt{2})$. Now $\mathbb{P}\left(\mathbf{v}^{3}, 0, \Omega\left(\mathbf{v}^{3}\right)\right)$ is connected so that $\mathbb{P}(\mathbf{v}, 0, \Omega(\mathbf{v}))$ is connected.

In order to effectively determine whether $\mathbb{P}(\mathbf{v}, 0, \Omega(\mathbf{v}))$ is connected, in addition to Th. 21, we still need a way to decide whether the fully subtractive algorithm will eventually reach some $\mathcal{K}_{d}$. At present, we are not even able to decide whether a given vector $\mathbf{v} \in \mathbb{R}^{d}$ belongs to $\mathcal{K}_{d}$. This lets some open questions and some research directions. Given $\mathbf{v} \in \mathbb{R}^{d} \backslash\{0\}$ we are interested in the following questions:

- decide whether OFS will erase some coordinate, i.e. whether $v_{1}^{n}=0$ for some $n$;

- if not, decide whether $\mathbf{v}$ belongs to $\mathcal{K}_{d}$;

- if $\mathbf{v} \in \mathcal{K}_{d}$, characterise the values of $\mu$ for which $\mathbb{P}(\mathbf{v}, \mu, \Omega(\mathbf{v}))$ is connected.

\section{References}

1. Andrès, E., Acharya, R., Sibata, C.: Discrete analytical hyperplanes. CVGIP: Graphical Model and Image Processing 59(5), 302-309 (1997)

2. Berthé, V., Domenjoud, E., Jamet, D., Provençal, X.: Fully subtractive algorithm, tribonacci numeration and connectedness of discrete planes. RIMS Lecture notes 'Kokyuroku Bessatu' (to appear, 2014)

3. Berthé, V., Jamet, D., Jolivet, T., Provençal, X.: Critical connectedness of thin arithmetical discrete planes. In: Gonzalez-Diaz, R., Jimenez, M.-J., Medrano, B. (eds.) DGCI 2013. LNCS, vol. 7749, pp. 107-118. Springer, Heidelberg (2013)

4. Brimkov, V.E., Barneva, R.P.: Connectivity of discrete planes. Theor. Comput. Sci. 319(1-3), 203-227 (2004)

5. Domenjoud, E., Jamet, D., Toutant, J.-L.: On the connecting thickness of arithmetical discrete planes - extended version (in preparation, 2014)

6. Domenjoud, E., Jamet, D., Toutant, J.-L.: On the connecting thickness of arithmetical discrete planes. In: Brlek, S., Reutenauer, Ch., Provençal, X. (eds.) DGCI 2009. LNCS, vol. 5810, pp. 362-372. Springer, Heidelberg (2009)

7. Domenjoud, E., Vuillon, L.: Geometric Palindromic Closures. Uniform Distribution Theory $7(2)$, 109-140 (2012)

8. Jamet, D., Toutant, J.-L.: Minimal arithmetic thickness connecting discrete planes. Discrete Applied Mathematics 157(3), 500-509 (2009)

9. Kraaikamp, C., Meester, R.W.J.: Ergodic properties of a dynamical system arising from percolation theory. Ergodic Theory and Dynamical Systems 15(04), 653-661 (1995)

10. Meester, R.W.J.: An algorithm for calculating critical probabilities and percolation functions in percolation models defined by rotations. Ergodic Theory and Dynamical Systems 9, 495-509 (1989)

11. Réveillès, J.-P.: Géométrie discrète, calcul en nombres entiers et algorithmique. Thèse d'état, Université Louis Pasteur (Strasbourg, France) (1991)

12. Schweiger, F.: Multidimensional continued fractions. Oxford Science Publications. Oxford University Press, Oxford (2000) 